\title{
PROJETER LE RÉEL. NOTES SUR L'ATTITUDE CRITIQUE INHÉRENTE À LA NOTION DE RÉALISME, À PARTIR DU FILM CHUNG KUO, CINA DE MICHELANGELO ANTONIONI
}

\section{Stefanie Baumann}

Association Le Lisible et l'illisible | « Le Philosophoire »

2017/1 n 47 | pages 135 à 150

ISSN 1283-7091

ISBN 9782353380503

DOI 10.3917/phoir.047.0135

Article disponible en ligne à l'adresse :

https://www.cairn.info/revue-le-philosophoire-2017-1-page-135.htm

Distribution électronique Cairn.info pour Association Le Lisible et l'illisible.

(C) Association Le Lisible et l'illisible. Tous droits réservés pour tous pays.

La reproduction ou représentation de cet article, notamment par photocopie, n'est autorisée que dans les limites des conditions générales d'utilisation du site ou, le cas échéant, des conditions générales de la licence souscrite par votre établissement. Toute autre reproduction ou représentation, en tout ou partie, sous quelque forme et de quelque manière que ce soit, est interdite sauf accord préalable et écrit de l'éditeur, en dehors des cas prévus par la législation en vigueur en France. Il est précisé que son stockage dans une base de données est également interdit. 


\title{
Projeter le réel. Notes sur l'attitude critique inhérente à la notion de réalisme, à partir du film Chung Kuo, Cina de Michelangelo Antonioni
}

\section{Stefanie Baumann}

\begin{abstract}
Résumé
À sa sortie en 1973, le film documentaire Chung Kuo, Cina de Michelangelo Antonioni a provoqué des critiques farouches aussi bien en Chine qu'en Europe. Bien qu'elles se contredisent entre elles, ces objections se rejoignent toutes dans un noyau conceptuel autour de la notion de réalisme, entendu au sens marxiste comme représentation critique de la réalité. Cependant, en refusant d'accorder un sens cohérent et essentialiste à ses images, ce film permet de problématiser les présupposés de cette compréhension même du réalisme. Il préfigure ainsi certaines difficultés qui lui sont inhérentes et permet de concevoir une autre manière d'entendre la critique.
\end{abstract}

\section{Abstract}

Chung Kuo, Cina, a documentary film by Michelangelo Antonioni, aroused fierce criticism both in China and in Europe when it was released in 1973. While these objections contradicted one another, they all share a conceptual core : the notion of realism understood in a Marxian sense as a critical representation of reality. Yet by refusing to grant a coherent and essentialist meaning to its images, Chung Kuo, Cina problematizes the presuppositions of such an understanding of realism. In this way, the film anticipates some of its intrinsic difficulties and inaugurates a new form of criticism. 
T e documentaire Chung Kuo, Cina, de Michelangelo Antonioni, réalisé sur invitation du gouvernement chinois pour la télévision italienne (RAI) et tourné lors d'un voyage de cinq semaines en 1972, est aujourd'hui considéré comme un document rare sur la Chine en pleine Révolution Culturelle. De nos jours, les spectateurs sont surtout éblouis par la beauté de ces images montrant des gens dans leur quotidien ou faisant du Taï-Chi, de l'étrange poésie des rythmes et des mouvements, ou encore de la tendresse du regard du cinéaste auprès du peuple chinois qui est reflétée dans le film. Or, en 1973, quand ce film est sorti dans plusieurs pays occidentaux dont l'Italie et la France, il a suscité des critiques très violentes et des débats intenses des deux côtés du rideau de fer, dont les plus virulentes provenaient de la Chine elle-même. Ainsi a-t-on pu lire dans le Quotidien du peuple (Remim Ribao), à l'époque l'unique organe de presse officielle représentant le Comité Central du Parti Communiste Chinois accessible aux étrangers :

Le film antichinois LA CHINE, [...] traduit les sentiments de haine farouche que dans le monde actuel une poignée d'impérialistes et de sociaux-impérialistes éprouvent envers la Chine nouvelle. La sortie de ce film constitue un grave incident antichinois et une insolente provocation contre le peuple chinois. [...] [...] Hostile au peuple chinois, [Antonioni] a profité de cette occasion [son invitation à faire un film en Chine] pour recueillir spécialement, par des procédés douteux et méprisables, des matériaux destinés à calomnier et à attaquer la Chine pour parvenir à des fins inavouables. Ce film de trois heures et demie ne rend compte ni des choses nouvelles, ni de l'atmosphère et de la physionomie nouvelles de notre grande patrie, mais il a réuni une masse considérable de scènes et d'images malignement déformées pour attaquer les dirigeants de notre État, diffamer la Chine nouvelle spécialiste, calomnier notre Grande Révolution Culturelle Prolétarienne et déshonorer le peuple ${ }^{1}$.

Ce ton hostile, extrêmement méprisant, qui paraît aujourd'hui tellement déplacé étant donné la douceur et la poésie des images du film, reflète pourtant une problématique très complexe qui est liée à sa portée politique. Celle-ci devient plus manifeste une fois que l'on considère le contexte particulier dans lequel cette attaque intervient. En 1972, la Chine étant alors encore en pleine Révolution Culturelle,

1. Commentateur du Renim Ribao, Intention perfide et procédé méprisable. Critique du film antichinois tourné par Antonioni et intitulé "La Chine », Pékin, Éditions en langues étrangères, 1974. 
Antonioni venait visiter et filmer le pays à l'invitation du premier ministre Zou Enlai, dans le cadre d'une campagne d'ouverture de la Chine qui avait pour but d'accroître sa visibilité et de renforcer les liens d'amitié avec certains pays bienveillants. Les dirigeants du Parti Communiste Chinois croyaient avoir confié la tâche de représenter le nouveau visage socialiste de leur pays à un artiste de gauche du même bord, sensible à leur orientation politique et engagé pour leur cause ${ }^{2}$. Mais après la sortie du film, ce dernier s'est retrouvé rapidement au centre d'un combat politique à l'intérieur de la Chine, opposant la Bande des Quatre, donc l'aile radicale, "puriste », du parti maoïste, et Zou Enlai, plus modéré. Chung Kuo, Cina fut dans ce contexte une des œuvres instrumentalisées contre la tendance libérale de Zou Enlai : il représentait désormais les tendances antirévolutionnaires, voire même antichinoises, et devint l'objet d'une campagne de protestation de masse. Suite à cela, le film fut interdit en Chine, et Antonioni devint persona non grata ${ }^{3}$.

\section{Attitudes critiques en Chine et en France}

Les critiques chinoises comme celles que nous avons citées plus haut, acquièrent ainsi une détermination supplémentaire : elles relèvent d'une lutte idéologique qui cherchait à instrumentaliser certaines œuvres d'art en vue d'une politique particulière et partisane de la représentation. Ainsi, Antonioni est blâmé de ne pas avoir rendu une image juste de la Chine révolutionnaire - à savoir, une image héroïque, progressiste, optimiste, conforme au programme du réalisme socialiste

2. Voir par exemple A. Kerlan, « La Chine d'Antonioni », Vingtième Siècle, n 105, janvier-mars 2010, p. 342.

3. Voir par exemple X. Liu, « China's Reception of Michelangelo Antonioni’s Chung Kuo ", Journal of Italian Cinema \& Media Studies, vol. 2, n 1, mars 2014 ; A. Xiang, “"When Ordinary Seeing Fails': Reclaiming the Art of Documentary in Michelangelo Antonioni's 1972 China Film Chung Kuo", Senses of Cinema, n 67, juillet 2013, accessible en ligne: http://sensesofcinema.com/2013/feature-articles/when-ordinaryseeing-fails-reclaiming-the-art-of-documentary-in-michelangelo-antonionis-1972-chinafilm-chung-kuo ; ainsi que D. Edwards, "Looking at/Looking in Antonioni’s Chung Kuo, Cina: A Critical Reflection Across Three Viewings", Senses of Cinema, $\mathrm{n}^{\circ} 74$, mars 2015, accessible en ligne : http://sensesofcinema.com/2015/feature-articles/looking-at-lookingin-antonionis-chung-kuo-cina-a-critical-reflection-across-three-viewings/; (les deux consultés le 3 décembre 2016). 
dicté par le régime maoïste ${ }^{4}$. Au lieu de s'aligner avec l'imagerie officielle, montrant des masses de prolétaires en pleine action, des réussites matérielles et sociales de la Grande Révolution Culturelle ainsi que le personnage de son leader, Mao lui-même, dans des couleurs et des tons vifs et optimistes, le film d'Antonioni montre des scènes quotidiennes, le visage parfois morne des personnes qu'il croise lors de son voyage, des gestes et des objets qui relèvent de la vie ordinaire. Selon les critiques chinois de l'époque, loin d'être réaliste à leur sens du terme, ces tableaux anodins donnent une image pitoyable de la Chine : des vies misérables, des conditions pauvres, une lassitude à grande échelle - en somme: Antonioni aurait composé cette image d'une Chine indigente et malheureuse afin de donner raison à ses ennemis impérialistes.

De l'autre côté du rideau de fer, les critiques européennes du film d'Antonioni étaient certes moins dévastatrices. Mais elles mettaient également en cause son attitude apolitique, en particulier son refus de prendre une position claire, tout en blâmant justement son apparent manque d'esprit critique. Ainsi, le sinologue Simon Leys, qui dénonçait depuis un moment le régime maoïste et la Grande Révolution Culturelle comme machine de propagande sanglante au service du pouvoir, décrit le film d'Antonioni comme l' « œuvrette» d'un «farceur» sans intérêt $^{5}$ : selon lui, Antonioni n'aurait tout simplement pas compris ce que la situation réelle lui cachait. Ignorant les enjeux de pouvoir et les répressions politiques qui régnaient en Chine à ce moment, son film ne révélerait que l'incapacité même de son auteur à prendre une posture critique adéquate. Lui aussi reproche donc à Antonioni son défaut de réalisme, mais cette fois-ci, parce qu'il n'aurait pas réussi à capter la réalité à cause de sa naïveté qui l'aurait menée à se laisser emporter par des projections romantiques ou utopiques d'une Chine qui n'existait pas. Selon Leys, Antonioni n'aurait donc pas su construire le «sens véritable » dissimulé sous le masque de la propagande maoïste.

Certains intellectuels de gauche, notamment Jacques Aumont et Serge Daney, alors auteurs pour les Cahiers du cinéma, déploraient surtout l'aspect prétendument neutre exprimé à travers le style, les motifs et les techniques de montage pour lesquels Antonioni a opté, donc

4. Voir aussi J. Lin, "Seeing a World apart. Visual Reality in Michelangelo Antonioni's Chung Kuo/Cina", ARTMargins vol. 3, octobre 2014, p. 21-44.

5. S. Leys, «Images brisées », in Essais sur la Chine, Paris, Éditions Robert Laffond, 1998, p. 518. 
son approche apparemment apolitique, acritique et superficielle ${ }^{6}$. Selon Daney, des images montrant une réalité hautement politisée comme celle de la Chine à ce moment historique, relèvent inéluctablement du politique et appellent donc à une critique précise ${ }^{7}$. Prétendre à une neutralité en produisant des images à tendance «naturalistes » serait méconnaître non seulement leur nature, mais aussi la portée qui leur est fatalement inhérente. "Il n'y a pas au cinéma que de la rencontre, du naturel, du comme par hasard, écrit-il dans un article, il n'y a pas d'image qui ne vise sournoisement (naturalisme) ou explicitement (publicité) à devenir une image de marque, c'est-à-dire du figé, du bloqué, du répressif. Et nous ajoutons : le typage sournois qui sourd de Chung Kuo n'est pas sans arrière-pensées ni malveillance $»^{8}$. La démarche et l'attitude d'Antonioni telles que médiatisées par son film occulteraient la réalité rencontrée en la considérant comme nature, comme donnée objective simplement là, alors qu' «[u]ne caméra et un magnétophone qui se branchent naïvement sur la réalité chinoise rencontrent nécessairement [une] pré-mise en scène sociale. Soit elle la reconduira (en la faisant passer comme spontanée), soit elle la fera oublier un instant (mais alors, il faut morceler). Le naturalisme est une technique qui reconduit quelque chose qui lui préexiste : la société en tant qu'elle est déjà une mise en scène. Travailler ce donné, casser cette pré-mise en scène, la rendre visible en tant que telle, est toujours une entreprise courageuse, difficile, impopulaire. Le réalisme est toujours à gagner $»^{9}$.

6. Voir S. Daney, «La remise en scène », Cahiers du cinéma, ${ }^{\circ} 268$, juillet 1976, reproduit dans La Rampe, Paris, Éditions Gallimard, 1983, p. 54-63, et J. Aumont, «La Chine (M. Antonioni) », Cahiers du Cinéma, n 248, 1973.

7. Serge Daney reprend cette critique dans d'autres articles comme « Une auberge espagnole singulièrement silencieuse. Michelangelo Antonioni, La Chine (Chung Kuo) », Libération, 4 octobre 1973, repris dans La maison cinéma et le monde. 1. Le Temps des Cahiers 1962-1981, Paris, P.O.L., 2001, p. 145-148, et «Fonction Critique 1 », Cahiers du Cinéma, $\mathrm{n}^{\circ} 248$, septembre-octobre 1973, repris dans La maison cinéma et le monde. 1. Le Temps des Cahiers 1962-1981, p. 315-317 et «Fonction critique 2 », Cahiers du cinéma, $\mathrm{n}^{\circ} 249$, février-mars 1974 , repris dans La maison cinéma et le monde. 1. Le Temps des Cahiers 1962-1981, op. cit., p. 318-323.

8. Serge Daney, « La remise en scène », in La Rampe, op. cit., p. 56.

9. Ibid., p. 61. 


\section{Spectres du réalisme critique}

Toutes ces critiques, si différentes et opposées qu'elles soient, se rejoignent en un noyau conceptuel central, plus précisément dans le spectre conflictuel de ce que l'on peut appeler réalisme. Or, cette notion qui se rapporte dans un sens large au réel socio-politique, en d'autres termes à ce qui est « objectif» au sens marxiste-léniniste du terme, et à sa représentation adéquate, semble admettre plusieurs interprétations bien différentes. Toujours est-il que le terme de réalisme, dans toutes ses formes, implique celui de critique, au sens d'une problématisation de ce qui n'est qu'apparent. Déceler le dispositif à travers lequel s'agence l'omission de la réalité véritable, à condition que celle-ci soit saisie conceptuellement et défendue par celui qui s'y engage, constitue le nœud de cette critique.

L'imagerie révolutionnaire optimiste telle que requise par le réalisme socialiste héroïque reflète l'intention de faire ressortir l'image d'une société prolétarienne en germination, en donnant une visibilité aux ouvriers et aux transformations progressistes en cours. L'image n'est donc pas censée reproduire la réalité telle qu'elle apparaît, mais représenter positivement une réalité in nuce, qui serait en train de fleurir ou qui serait encore à réaliser. Le concept de réalité même serait donc à penser comme étant essentiellement structurel et idéel. Par conséquent, sa représentation serait censée non pas reproduire, mais construire une image adéquate à cette essence, c'est-à-dire, selon les partisans du réalisme héroïque chinois, au service de la politique et contribuant à la construction de l'État communiste en enflammant, par cette vision triomphaliste, l'enthousiasme de ceux qui seraient encore sceptiques.

Mais il existe un autre réalisme qui est très différent du premier bien qu'il s'appuie également sur une conception marxiste de la réalité et de la critique qui lui serait appropriée : au lieu d'esthétiser la politique en cours pour la soutenir, afin qu'elle puisse s'accomplir pleinement, au lieu de forger une imagerie de ses exploits révolutionnaires pour les consolider, le réalisme dont parle par exemple Serge Daney est au contraire censé déconstruire l'apparence immédiate de cette réalité, et construire du sens à travers des moyens artistiques propres. Cette idée d'un réalisme délibérément critique, politique, voire militant, renvoie à plusieurs égards au débat autour de l'expressionnisme publié dans le journal d'exilés Das Wort, qui a opposé dans les années 1930 des intellectuels tel que Georg Lukács, Ernst Bloch et Bertold Brecht, 
entre autres ${ }^{10}$. Recourant tous à un concept critique de réalisme comme instrument de lutte contre le nazisme et le capitalisme, donc au service de l'émancipation, les contributeurs en tirent pourtant des conclusions différentes quant à l'attitude de l'auteur, le rapport entre contenu et forme dans l'œuvre et la position du spectateur.

Dans sa conception du réalisme, Georg Lukács part de la théorie du reflet conçue par Marx et Engels et reprise plus tard par Lénine. Pour cette théorie, la production culturelle, en tant que manifestation de la superstructure, est déterminée en dernière instance par la base, c'est-àdire par les conditions socio-économiques et politiques dans lesquelles elle s'inscrit nécessairement, bien que ces deux soient médiatisées l'une par l'autre ${ }^{11}$. Selon Lukács, les productions culturelles réalistes, comme les grands romans balzaciens du XIX ${ }^{\mathrm{e}}$ siècle, arrivent à rendre perceptibles et intelligibles les soubassements déterminants de la réalité, en représentant une situation particulière dans une société en tant que totalité ${ }^{12}$. Le particulier s'exprime à travers les personnages, qui sont conçus en tant que «types ». Le particulier est donc directement rapporté à l'universel, extériorisant ainsi la dialectique réelle, objective : «Dans le grand réalisme [...], se trouve figurée une tendance de la réalité non immédiatement évidente mais objectivement d'autant plus durable et importante, à savoir l'homme dans ses multiples relations avec la réalité et précisément, en l'occurrence, ce qu'il y a de durable

10. La quasi-totalité de ce débat a été publié en allemand sous le titre Die Expressionismusdebatte. Materialien zu einer marxistischen Realismuskonzeption, H.-J. Schmitt (éd.), Frankfurt am Main, Édition Suhrkamp, 1978, en anglais, Frederic Jameson a rassemblé quelques textes clés de ce débat sous le titre Aesthetics and Politics, Verso Books, Londres, 1973. En français, pour les contributions de Lukács, voir G. Lukács, Problèmes du réalisme, Paris, L’Arche, 1975 ; pour Brecht, voir B. Brecht, Sur le réalisme. Précédé de Art et politique et Considérations sur les arts plastiques, Paris, L'Arche, 1970.

11. G. Lukács est évidemment un penseur marxiste très complexe et sa pensée ne peut être réduite à un économisme suivant le schéma simpliste d'une base conditionnant unilatéralement la superstructure, schéma qu'il transforme d'ailleurs dans son magistral Histoire et conscience de classe, en réintroduisant la catégorie hégélienne de la totalité.

12. «Si la littérature est effectivement une forme particulière du reflet de la réalité objective, il lui importe beaucoup d'appréhender cette réalité telle qu'elle est effectivement constituée et ne peut se borner à reproduire l'immédiateté des phénomènes. Si l'écrivain vise à une appréhension et à une représentation de la réalité telle qu'elle est effectivement, c'est-à-dire s'il est vraiment réaliste, le problème de la totalité objective jouera un rôle décisif [...] », G. Lukács, « Il y va du réalisme », in Problèmes du réalisme, op. cit., p. 248. 
dans cette riche multiplicité ${ }^{13}$. Par conséquent, la forme, forcément, aspire à l'universel. Elle est pensée par rapport au contenu dialectique qu'elle est censée exprimer ou, pour le dire autrement, elle devient en quelque sorte le support de la vérité sur le réel qu'elle donne à comprendre. Dans cette variante du réalisme, les images immédiates qui apparaissent doivent donc être sélectionnées et orchestrées par l'artiste afin que leur articulation dans l'œuvre fasse ressortir la totalité qui n'est pas immédiatement perceptible, mais qui constitue le noyau réel. À cet égard, les images d'Antonioni seraient reléguées à ce que Hegel appelait « l'existence paresseuse ». Une existence bien là, mais comme elle ne participe pas à la dynamique historique, elle n'a pas de réalité en tant que telle, n'est pas wirklich. Et il est nécessaire de comprendre ces positions réalistes, qui de prime abord se donnent comme ayant peu à voir avec le réel, en rapport avec la réalité au sens de Wirklichkeit : ce qui a de l'effet et qui s'effectue. En dévoilant la totalité dans sa nécessité, ce qui devient saisissable serait la tendance en train de se réaliser dans sa nécessité, et c'est cela qui devrait gagner les sceptiques à la cause socialiste. D'une certaine manière, cette perspective englobe la précédente puisqu'elle est tout autant au service de la politique.

Or, elle reste contemplative. C'est précisément une des critiques formulées par Bertolt Brecht. Contre le réalisme lukácsien, qui met le public des œuvres dans une position de contemplation passive (la critique sourd d'une totalité qui lui est donnée à voir), Brecht défend un réalisme de la construction basé sur le principe de distanciation (Verfremdung/V-Effekt). Celui-ci est employé afin de permettre une prise de conscience active du médium même par lequel la critique du réel est effectuée. Le médium devient ainsi lui-même objet de la critique, en même temps que la position du spectateur perd son évidence. La critique brechtienne vise donc à la fois le rapport entre la forme et le contenu et la conception sous-jacente du réel même. Car, selon Brecht, les logiques de la perception se transforment tout comme la réalité. Recourir à l'idée d'une totalité dans la représentation de la réalité crée une idée fausse de cette dernière, car celle-ci, devenue fondamentalement fragmentaire, n'est plus perceptible ou même concevable en tant que totalité. Toute tentative de forger l'image d'une totalité serait donc à critiquer tout comme l'idéologie qui lui est sous-jacente. Ainsi, il faudrait inventer des formes nouvelles adaptées à la situation actuelle qui empêchent le spectateur ou lecteur de se distraire avec l'illusion d'un monde qui n'est

13. G. Lukács, « Il y va du réalisme », op. cit., p. 262. 
plus. Brecht reproche à Lukács de s'attacher à des formes anciennes incapables de capter et de critiquer l'actualité de manière adéquate. Pour être réaliste, l'artiste ne devrait donc pas suivre un modèle préétabli, mais construire à même la réalité une œuvre capable d'ouvrir vers un regard critique, en pensant la forme avec le contenu. La notion de réalité « objective », celle qui se profile une fois que ses conditions idéologiques, politiques, socioéconomiques sont prises en compte dans sa construction, est ici pensée et travaillée artistiquement de manière critique, transformatrice, voire parfois militante. Ce réalisme-ci entend donc contribuer activement aux transformations d'une société par la mobilisation du public, qui est alors censé penser à même les œuvres et s'engager pour la bonne cause.

Toujours est-il que tous ces réalismes reposent, d'une manière ou d'une autre, sur une analyse de la réalité en question - analyse qui servirait de fondement pour l'élaboration de la critique. Cette analyse préexiste jusqu'à un certain point à l'œuvre, conduit son auteur à prendre certaines décisions politiques et à faire certains choix esthétiques afin de dégager les enjeux complexes constitutifs de la réalité en question. En définissant ce qui serait l'essentiel à saisir d'une société, l'auteur d'une telle œuvre réaliste prend ainsi une position savante, didactique, engagée, ou encore idéologique : il veut nous donner à percevoir, à comprendre sensiblement et intelligiblement, à penser, un tableau de la réalité déjà dessiné. Il ne nous laisse pas seuls avec les images qu'il donne à voir, mais suggère ou impose un chemin censé nous aider à l'interpréter. Au-delà de la question de savoir si l'art doit être engagé, et comment il doit le faire, ce qui transparaît ici, c'est un certain autoritarisme de toutes ces positions réalistes. Et n'est-il pas vrai que la Révolution Culturelle a cherché, au moins était-ce son utopie fondamentale, justement à défaire cet autoritarisme ? En ce sens, le film d'Antonioni suit, si ce n'est la lettre, du moins l'esprit de la Révolution Culturelle.

\section{Regarder en tant qu'étranger : Antonioni en Chine}

Le parti pris d'Antonioni est bien différent de ces positions réalistes. Chung Kuo, Cina est un film qui ne veut pas faire ressortir l'essentiel de la réalité qu'il rencontre, il ne nous montre pas une réalité en tant que sujet d'analyse qu'il examinerait de manière critique afin de déceler ses rouages. Le film d'Antonioni ne montre explicitement 
qu'un regard, celui d'un étranger, vers une réalité qui lui demeure étrangère : "Ces cinq semaines n'ont permis qu'un regard furtif; en tant que voyageur, j'ai vu les choses avec un œil de voyageur, écritil. J'ai essayé d'emmener le spectateur avec moi, de le prendre par la main pour ainsi dire, et de le laisser m'accompagner dans ce voyage. De plus, les structures sociales et politiques sont des entités abstraites qui ne se laissent pas facilement exprimer par les images. Il aurait fallu ajouter des mots à ces images, et ceci n'était pas mon rôle. Je ne suis pas allé en Chine pour la comprendre, mais seulement pour la voir. Pour la regarder et enregistrer ce qui s'est passé sous mes yeux $»^{14}$.

Chung Kuo, Cina, pose donc la question du rapport entre une réalité et sa représentation adéquate différemment, déjà parce que nous ne sommes pas seulement confrontés avec une seule réalité à comprendre - celle de la Chine maoïste - mais avec une intersection entre deux réalités, qui correspond ici à la réalité d'un voyageur allant à la rencontre d'une réalité qui résiste largement à l'assimilation de son regard. La réalité d'Antonioni est celle du touriste qui ne parle pas la langue du pays visité, qui n'est pas familier avec les codes et les conditions sociales et politiques - même s'il connaissait les discours occidentaux partisans ou hostiles de son temps, avec toutes les projections qu'ils véhiculaient. Loin d'occuper une position stable et souveraine, Antonioni rend explicite qu'il dépendait non seulement de ses guides et traducteurs, mais aussi des représentants officiels de la Chine et de sa bureaucratie qui ont limité les possibilités de déplacement de façon considérable. En outre, aucune parole chinoise n'est traduite dans le film, il ne donne donc pas la voix à ceux qui l'habitent : il reste toujours une part de mystère, d'étrangeté, qui ne disparaît pas tout au long du film. Même les commentaires d'Antonioni, qui par moments offrent des indications sur ce que l'on voit, ou plus précisément sur ce qu'il voit lui, prennent de la distance vis-à-vis de ce qu'il trouve sur place. Soucieux de rester subjectif, personnel, ses paroles accompagnent les images sans intention de les encastrer dans un récit objectif. Dans ce dispositif, la Chine demeure lointaine et en grande partie incompréhensible, non saisissable avec les outils conceptuels et artistiques du réalisateur. Ce qu'Antonioni capte avec sa caméra - il le

14. Michelangelo Antonioni dans "Talking to Michelangelo", The Guardian, 18 février 1975, repris dans M. Antonioni, The architecture of vision. Writings \& Interviews on Cinema, Chicago, University of Chicago Press, 2007, p. 327 (traduction de l'auteure). 
dit aussi explicitement dans son commentaire - ce sont « les Chinois ». Non la place Tian'anmen dans son immensité impressionnante, mais la manière dont elle est appropriée, vécue, habitée, un jour quelconque. Non pas les masses mobilisées, chorégraphiées, organisées, mais des personnes qui apparaissent dans une foule, en demeurant toujours des êtres singuliers, puisque Antonioni focalise son objectif sur leurs visages et leurs gestes particuliers.

Filmer ce qu'il voit, ce qui se présente à son regard en tant que réalité visuelle apparente est, pour Antonioni, "aussi une façon de faire du "cinéma vérité". Attribuer à quelqu'un son histoire, écrit-il, c'est-à-dire l'histoire qui coïncide avec son apparence, sa position, son poids, son volume dans un espace $\gg^{15}$. Antonioni filme des gens dans les rues, des restaurants populaires, des usines, des parcs ou des écoles, des villages pauvres et des villes en pleine expansion, en décalant souvent son objectif vers des scènes particulières qui sortent du grand récit, ouvrant une lucarne sur une certaine quotidienneté. Ce qui l'intéresse, ce sont les visages et les corps, leurs rythmes singuliers et collectifs, leurs manières de s'approprier l'espace, comme la lenteur des gestes fluides et gracieux des personnes pratiquant le Taï-Chi, la discipline des enfants qui dansent et leur jeu détendu pendant la pause, les marches militaires, la formation spontanée de groupes, mais aussi le calme étonnant qui règne pendant une césarienne sous acupuncture. Or, à travers ces scènes apparemment sereines, paisible, une autre réalité s'immisce à certains moments dans ce film, avec des effets politiques si puissants qu'ils ont débordé le cadre de l'écran jusque dans la violence des invectives dont Antonioni fut l'objet. Cette autre réalité témoigne en même temps d'un esprit critique autre qui serait à l'œuvre, de manière immanente, qui ne relève pas d'une prise de partie pour ou contre un système ou une idéologie, mais qui agit sensiblement à travers le film sur ceux qui le regardent.

Cependant, ce n'est pas en suscitant un effet de familiarisation avec les personnes que cette autre couche du réel transparaît. Antonioni ne cherche pas à produire une identification possible; tout demeure étranger, lointain, autre. Ceci devient très explicite dans ces longs moments où Antonioni filme des personnes qui lui rendent le regard. Dans plusieurs séquences, la caméra s'arrête sur un visage qui la regarde à son tour droit dans les yeux. La présence de la caméra et de

15. La Stampa, 11 juillet 1963, et Cinéma nuovo, $\mathrm{n}^{\circ} 167$, janvier-février 1964. Texte disponible dans le livre: M. Antonioni, Écrits, Paris, Images modernes, 2003, p. 65-67. 
l'étranger derrière elle reste donc très manifeste tout le long du film. Ainsi, le spectateur devant l'écran semble être pris au jeu, mis dans la situation de celui qui est filmé. En dépit de l'étrangeté infranchissable, il s'avère qu'Antonioni cherche à établir une « égalité du regard », pour citer un mot qu'utilise Chris Marker dans son film Sans Soleil. Il semble qu'un contact aurait lieu, un échange de regards curieux, mais distants et impénétrables qui engendre virtuellement, dans le dispositif filmique, une réciprocité particulière, qui ne fait que s'amorcer puisqu'elle ne peut s'accomplir en réalité. Cette rencontre entre deux regards, celui d'Antonioni à travers la caméra et celui de la personne filmée, ne se résume pas, il n'y a pas de signe de connivence ou d'entente implicite sur ce qui serait à critiquer ou à soutenir. Il s'agit plutôt d'une confrontation manquée qui court-circuite d'une certaine manière le dispositif ethnologique classique de celui qui regarde - activement, en essayant de saisir et de comprendre l'autre - et celui qui est regardé - passivement, telle une écriture à déchiffrer. Car ceux qui sont regardés résistent, avec les moyens pauvres qu'ils détiennent, en soutenant le regard de l'autre et en le lui rendant. L'étrangeté est mutuelle, et la longue durée des captations de ces regards a cet effet étrange : celles-ci déploient en quelque sorte l'altérité de l'autre, en déjouant les projections dont il est sujet. Elle demande non seulement de regarder plus, autrement, sans préjugés, mais elle remet aussi en question la légitimité de la situation même de celui qui regarde, l'évidence avec laquelle ce dispositif opère. Visiblement, il y a une certaine violence inhérente dans le fait que le réalisateur d'un film dit documentaire puisse choisir sa position face à l'autre et le transformer en motif, en utilisant son image pour son propos, tandis que les personnes filmées ne peuvent que résister muettement.

\section{Politiques de l'altérité}

Une des scènes du film fait remarquablement ressortir cette situation ambivalente et complexe. Tournée malgré l'interdiction des représentants chinois officiels dans un village qui, selon Antonioni, n'avait jamais été visité par un Occidental auparavant, elle consiste en une longue séquence dévisageant les villageois en train de fixer l'étranger du regard. Elle ressemble étrangement à une situation décrite par Julia Kristeva qui a fait le voyage en Chine avec le groupe Tel quel deux ans après Antonioni : "Tout le village est sur la place [...]. Une foule immense est assise sous le soleil : elle nous attend sans mot, sans 
mouvement. Des yeux calmes, même pas curieux, mais légèrement amusés ou anxieux, en tout cas perçants, et sûrs d'appartenir à une communauté avec laquelle nous n'aurons jamais rien à voir. [...] Comme s'ils découvraient des animaux bizarres et drôles, inoffensifs mais insensés. Sans agressivité, mais au-delà d'un abîme de temps, d'espace. [...] Je ne me sens pas étrangère comme à New York ou à Bagdad. Je me sens singe, martienne, autre ${ }^{16}$. À l'instar de Kristeva, qui dépeint, dans son essai sur les femmes chinoises, son expérience d'une altérité irréductible pour introduire une perspective qui échappe à l'universalisme, l'eurocentrisme, et l'humanisme français, Antonioni dit effectivement que l'étranger, c'est «nous»: lui en tant que réalisateur, mais aussi les spectateurs occidentaux du film. L'ambiguïté de l'entreprise de filmer cette réalité en dehors de l'itinéraire prévu, la violence de la captation par la caméra de villageois qui ne sont pas préparés à se voir transformés en représentation, d'une réalité qui n'attend pas d'intrus, deviennent très palpables dans cette scène.

Cependant, cette violence qui se fait sentir se reflète en deux sens : premièrement, il y a violence dans l'acte de filmer un groupe de personnes sans demander leur avis, deuxièmement il y a violence dans le fait que ces personnes n'étaient pas considérées - par le chef du village et par ceux qui ont planifié à l'avance l'itinéraire d'Antonioni -, comme étant présentables, comme dignes d'avoir une visibilité en dehors du village et de représenter la Chine. Ainsi, la politique maoïste entre en jeu par la porte de derrière, par le biais de la question de la visibilité. Comme nous l'avons déjà évoqué, la politique n'est jamais présentée frontalement, par une mise en scène directe des signes de pouvoir. Mais le rapport entre signes de l'idéologie maoïste et vécu n'est certainement pas absent dans le film, bien qu'il ne soit pas inscrit dans un discours qui dirigerait sa lecture et son sens. C'est le regard d'Antonioni, à travers lequel nous percevons la Chine, qui perce dans ce rapport en ne faisant aucune différence entre des images qui seraient « bonnes » à montrer - c'est-à-dire, conformes à l'idée à défendre - et d'autres qui seraient « mauvaises » - images équivoques, contrariantes, complexes. Le naturalisme antonionien, pour reprendre le mot de Serge Daney, consiste à voir des choses qui échappent à la désignation, qui ne font pas encore de sens, qui ne se laissent pas encore intégrer à une position stable. Tout en étant médiatisé par la position subjective

16. J. Kristeva, Des Chinoises, Éditions des Femmes, Paris, 1974. 
très présente du réalisateur, il fait ressortir la double présence de deux altérités qui se confrontent à travers sa caméra.

Ainsi, Chung Kuo/Cina n'exclut ni les images de propagande, ni celles qui les contrecarrent, et qui relèvent des habitudes, des gestes et des comportements. Pourtant, encore une fois Antonioni se garde de les approprier pour en faire une analyse ou en tirer une conclusion. En ce qui concerne les signes maoïstes perceptibles à travers le film, Antonioni écrit: "C'est de la propagande, mais ce n'est pas un mensonge $»^{17}$. La propagande est considérée comme partie intégrante de la réalité à laquelle il se voit confronté ; elle n'est pas son autre, mais s'inscrit également dans le paysage et dans les corps. Un deuxième effet politique est ainsi réalisé : l'effet de miroir que Antonioni tend à ses spectateurs. En frustrant leurs attentes, qu'elles soient celles d'une Chine triomphante considérée comme alternative à la situation européenne ou d'une Chine violente et dictatoriale, en refusant de lier les éléments du film autour d'une essence délimitée, face à laquelle on pourrait se décider à prendre une position déterminée, Antonioni fait exploser le dispositif de projection que l'Occident entretenait envers la Chine, qu'il soit pour la critiquer ou la hisser aux nues. Et c'est ce mouvement de ressac qui rejaillit sur les images et qui leur donne cette fraîcheur et cette poésie.

\section{Critiquer autrement}

Et c'est aussi cette absence de directive, cette ouverture qui garde le spectateur dans une position vague, qui constitue pour Roland Barthes la force de l'œuvre d'Antonioni : " [Q]uand vous déclarez (dans un entretien avec Godard): "J'éprouve le besoin d'exprimer la réalité dans des termes qui ne soient pas tout à fait réalistes", vous témoignez d'un sentiment juste du sens : vous ne l'imposez pas, mais vous ne l'abolissez pas. Cette dialectique donne à vos films [...] une grande subtilité : votre art consiste à toujours laisser la route du sens ouverte, et comme indécise, par scrupule. C'est en quoi vous accomplissez très précisément la tâche dont notre temps a besoin : ni dogmatique, ni insignifiant $\gg{ }^{18}$. Au lieu de faire comprendre, de commenter de manière

17. M. Antonioni, "Is it still possible to film a documentary?", in The architecture of vision. Writings \& Interviews on Cinema, op. cit., p. 110 (trad. de l'auteure).

18. R. Barthes, "Cher Antonioni », Cahiers du cinéma, n³11, mai 1980, p. 10. 
critique, d'orienter le spectateur, l'opération en cours dans Chung Kuo, Cina, consisterait donc à déployer ce qui se dessine en tant qu'étant là, devant l'objectif, afin d'ouvrir vers de nouveaux questionnements qui ne se sont pas encore clairement délimités, qui ne sont peut-être pas encore énonçables, mais qui s'immiscent déjà dans les plis de l'image avec une force critique qui s'articule sensiblement. Pour sortir du cliché, pour ouvrir la voie à un regard intensif, Antonioni prolonge la durée pendant laquelle sa caméra regarde une personne, une situation, un objet, un paysage. Antonioni refuse de prendre parti, de construire une position réaliste au sens lukácien, brechtien ou godardien du mot, peutêtre par pudeur, parce qu'il est conscient qu'il n'en a pas les moyens, ou encore parce qu'il comprend sa tâche différemment: comme la recherche d'une position d'auteur à la fois intéressée et non-autoritaire, autoréflexive, descriptive, et ouverte aux contradictions. C'est ainsi qu'une nouvelle manière de concevoir l'esprit critique se dessine. «Critique, écrit Jacques Rancière, est l'art qui déplace les lignes de séparation, qui met de la séparation dans le tissu consensuel du réel, et, pour cela même, brouille les lignes de séparation qui configurent le champ consensuel du donné $[\ldots] »^{19}$. Chez Antonioni, c'est la frontière entre ici et là, entre bonnes et mauvaises images, entre idées reçues et analyses complexes, qui se brouille tout en restant présente, mais qui résiste à se faire instrumentaliser.

\section{Bibliographie}

Antonioni M., Écrits, Paris, Images modernes, 2003.

-, The architecture of vision. Writings \& Interviews on Cinema, Chicago, University of Chicago Press, 2007.

Aumont J., La Chine (M.Antonioni) in Cahiers du Cinéma, 248, 1973.

Barthes R., Cher Antonioni in Cahiers du cinéma 311, mai 1980.

Brecht B., Sur le réalisme. Précédé de Art et politique et Considérations sur les arts plastiques, Paris, L'Arche, 1970.

Daney S., La Rampe, Paris, Éditions Gallimard, 1983.

_, La maison cinéma et le monde. 1. Le Temps des Cahiers 1962-1981, Paris, POL, 2001.

EDWARDs D., "Looking at/Looking in Antonioni's Chung Kuo, Cina: A Critical

Reflection Across Three Viewings", Senses of Cinema, n 74, mars 2015,

19. J. Rancière, «Les paradoxes de l'art critique », in Le spectateur émancipé, Paris, La Fabrique, 2008, p. 85. 
accessible en ligne: http://sensesofcinema.com/2015/feature-articles/ looking-at-looking-in-antonionis-chung-kuo-cina-a-critical-reflectionacross-three-viewings/.

Jameson F. (éd.), Aesthetics and Politics, Londres, Verso books, 1973.

Kerlan A., « La Chine d'Antonioni », Vingtième Siècle, n 105, 2010.

Kristeva J., Des Chinoises, Paris, Éditions des Femmes, 1974.

Leys S., Essais sur la Chine, Paris, Robert Laffond, 1998.

LiN J., "Seeing a World apart. Visual Reality in Michelangelo Antonioni's Chung Kuo/Cina", ARTMargins, vol. 3, octobre 2014, p. 21-44.

Liu X., "China's Reception of Michelangelo Antonioni's Chung Kuo », in Journal of Italian Cinema \& Media Studies, vol. 2, n 1, mars 2014.

LuKács G., Problèmes du réalisme, Paris, L'Arche, 1975.

RANCIĖRE J., Le spectateur émancipé, Paris, La Fabrique, 2008.

Renim RibaO, Intention perfide et procédé méprisable. Critique du film antichinois tourné par Antonioni et intitulé «La Chine », Pékin, Editions en langues étrangères, 1974.

Sснмiтt H.-J. (éd.), Die Expressionismusdebatte. Materialien zu einer marxistischen Realismuskonzeption, Frankfurt am Main, Suhrkamp, 1978.

XIANG A., "'When Ordinary Seeing Fails': Reclaiming the Art of Documentary in Michelangelo Antonioni's 1972 China Film Chung Kuo", Senses of Cinema, $\mathrm{n}^{\circ}$ 67, juillet 2013, accessible en ligne : http://sensesofcinema. com/2013/feature-articles/when-ordinary-seeing-fails-reclaiming-the-artof-documentary-in-michelangelo-antonionis-1972-china-film-chung-kuo. 